\title{
Optimization of Steering System of Forklift Vehicle for Idle Performance
}

\author{
Yuan Shen, ${ }^{1,2}$ Biao Chu, ${ }^{2}$ DongCai Liu, ${ }^{1}$ and Chang'an $\mathrm{Zhu}^{2}$ \\ ${ }^{1}$ School of Electronics and Information Engineering, Hefei Normal University, Hefei 230000, China \\ ${ }^{2}$ Department of Precision Machinery and Precision Instrumentation, University of Science and Technology of China, \\ Hefei 230027, China
}

Correspondence should be addressed to Yuan Shen; shenyuan@ustc.edu.cn

Received 28 July 2015; Revised 15 October 2015; Accepted 15 October 2015

Academic Editor: Milan Hladík

Copyright (C) 2015 Yuan Shen et al. This is an open access article distributed under the Creative Commons Attribution License, which permits unrestricted use, distribution, and reproduction in any medium, provided the original work is properly cited.

\begin{abstract}
This paper presents an optimal design process for the steering system of a forklift vehicle. An efficient procedure for minimizing the engine-induced idle vibration is developed in this study. Reciprocating unbalance and gas pressure torque as two major sources of engine excitation are studied. Using the field vibration tests and FEM analysis, the cause and characteristics of steering system's idle vibration are recognized. So as to distribute the characteristic modes based on the optimization strategy, global sensitivity analysis of the main parameters is also carried out to achieve the optimal combination of the optimization factors. Based on all analysis above, some structure modifications for optimization are presented to control the idle vibration. The effectiveness and rationality of the improvements are also verified through experimental prototyping testing. This study also makes it possible to provide a design guideline using CAE (computer aided engineering) analysis for some other objects.
\end{abstract}

\section{Introduction}

As multifunctional and versatile material transportation and handling equipment, forklifts are becoming widely used all around the world with the rapid development of logistics industry. Forklift industry has entered a period of rapid development, of which research and design levels are also increasing, while the vibration problem becomes a general item to measure the equipment quality. When operating the forklift, drivers perceive vibrations through the floor panel, the pedals, the gearshift lever, the seat, and the steering wheel. As a major occupational vibration exposure, more attention should be paid to Hand-Arm Vibration (HAV) where the energy enters the body through the hands of the operator $[1,2]$, especially for steering vibration, when the vibration is sensed by the driver through steering wheel, which directly influences drivers' operation. Severe vibration greatly reduces the worker's comfort, leading to a series of problems. The effects can include muscular fatigue, hands numbness, degraded circulatory functioning, and headaches [3].

Vibration problem can be improved from the following three aspects: suppression of the vibration excitation, improvement of the vibration transmission path, and optimization of the mechanical parameters. Kim et al. [2] described an analysis methodology of an optimal design process for a steering column system and supporting system while reducing the idle vibration sensitivity, which was expected to reduce the overall development period and number of proto tests. Shi et al. [4] used vibration tests and modal analysis method to recognize the cause of steering wheel's idle shaking, structural optimization, and lightweight design that were taken to solve the problem. Shariyat and Djamshidi [5] adopted DOE-based response surface methodology and sensitivity $\mathrm{NVH}$ analysis results of a full vehicle model in order to minimize the engine-induced harshness. M. L. $\mathrm{Xu}$ and G. N. Xu [6] improved the forklift vehicle system structure based on the multi-rigid-body dynamic method and efficiently decrease vibration energy and the fundamental frequency of primary structure is lower. Parker et al. [7] insisted vibration character of the steering system can be divided into two parts: the steering column system and the supporting structure; the optimal design of each part can be separately managed based on research of the main structural parameters. Kim et al. [8] described a development process 


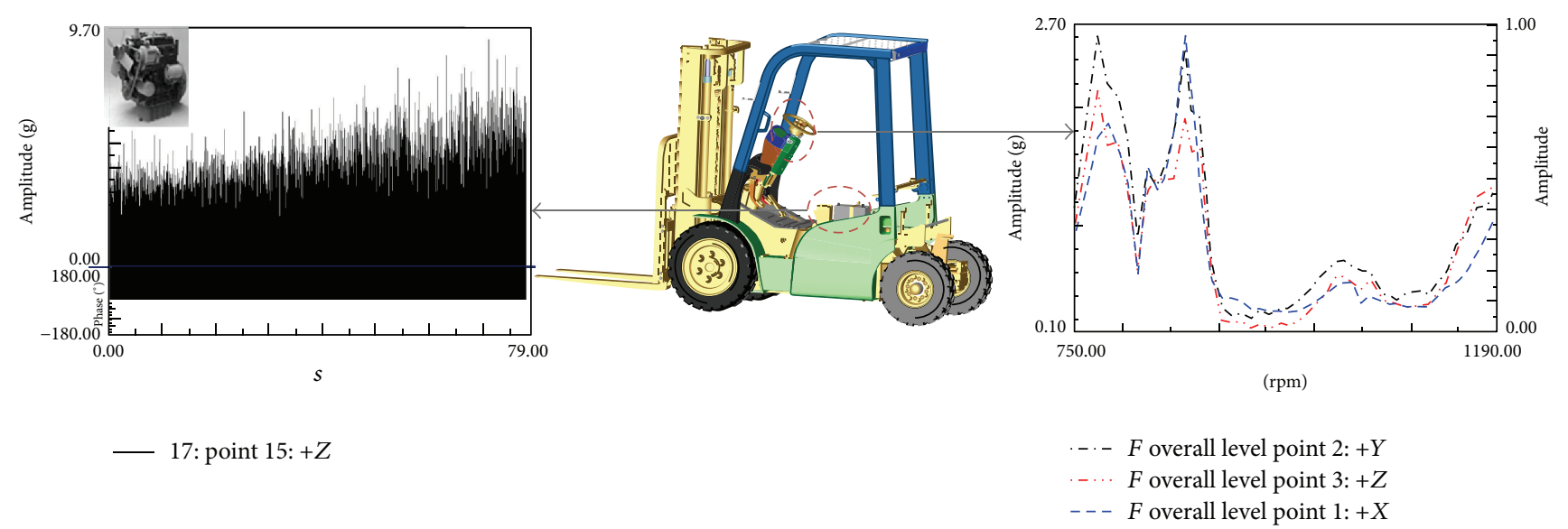

FIgURE 1: Technological process of the study.

of a high stiffness and lightweight vehicle for vibration performance; through the data level of body stiffness, the research efficiently shortened the development period by the stiffness analysis process. Also, some works that focus on the engine-mounting system optimization were developed by Royston and Singh $[9,10]$; the dynamic characteristics of the mounting system were changed so as to enhance the vibration isolation performance. Jia et al. [11] analyzed the vibration transmission path based on the vibration measurement technology; through the optimization of the mounting system, comfort of the vehicle is improved.

The objective of this paper is to suppress the vibration of forklift vehicles, by finding the vibration principle, providing advanced design and optimization direction. Among all studies mentioned before, most focus on the passenger cars, ignoring the dynamics performance of engineering machinery. In this study, optimization of the steering system of a forklift vehicle (to minimize the transmitted engine-induced vibration under idle condition) is introduced. The idle vibration analysis relies on the FEM analysis of the steering system, combined with feature extraction of vibration signals through field tests. The optimal combination of structural parameters of the steering system is rationally found so that the natural frequency distribution is optimal and the optimal vibration characteristic is obtained.

\section{Analysis of Engine Idle Loads}

While the forklift vehicle is working under idle operation, the only vibrational excitation is internal combustion inline engine as shown in Figure 1, and the major sources for idle shake are reciprocating unbalance inertia forces and gas pressure torque oscillations due to firing pulses and inertia of the rotating and reciprocating parts (piston, connecting rod, and crankshaft). The dynamic unbalance law of the cylinder piston crank mechanism is playing an important role in the design and improvement of the forklift vehicle.

2.1. Mass Unbalance Forces. The single cylinder piston crank mechanism is shown in Figure 2. It consists of three parts: a crank, a connecting rod, and a piston.

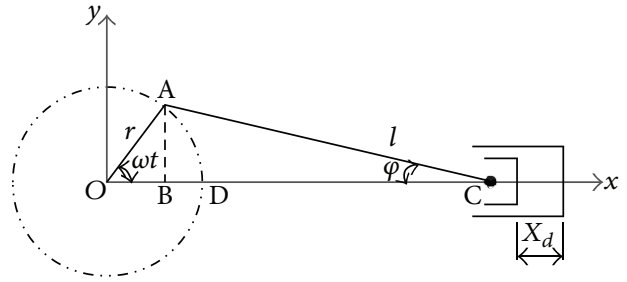

FIGURE 2: Crank mechanism of diesel engine, where $r$ is crank length; $l$ is connecting rod length; $\varphi$ is angle between piston centerline and connecting rod; $\theta=\omega t$ is crank angle from top dead center at time; $X_{d}$ is displacement of piston from top position.

Assume that the crank rotates with a constant angular velocity and the piston displacement $X_{d}$ can be expressed as

$$
X_{d}(t)=r(1-\cos \theta)+l(1-\cos \varphi) .
$$

According to the geometrical relationship

$$
\cos \varphi=\sqrt{1-r^{2} \cdot \frac{\sin ^{2} \theta}{l^{2}}} .
$$

So $X_{d}$ can be exactly described in terms of crank angle $\theta$,

$$
X_{d}=r(1-\cos \theta)+l\left(1-\sqrt{1-\frac{r^{2}}{l^{2}} \sin ^{2} \theta}\right),
$$

where $r / l$ is typically between $1 / 5$ and $1 / 3$. For a mathematical expression like $\sqrt{1-x}$ while $x \ll 1$, the expression can be simplified as $1-x / 2$ through Taylor series expansion, and the equivalent expression for $X_{d}$ turns out to be

$$
X_{d}=r(1-\cos \theta)+r^{2} \cdot \frac{\sin ^{2} \theta}{2 l} \text {. }
$$

The approximate piston displacement is as follows:

$$
X_{d}=\left(r+\frac{r^{2}}{4 l}\right)-r\left(\cos \theta+\frac{r}{4 l} \cos 2 \theta\right) .
$$


Then velocity and acceleration of the piston can be calculated by differentiation of the displacement of the piston with respect to time,

$$
\begin{aligned}
& V_{d}=\omega r\left(\sin \theta+\frac{r}{2 l} \sin 2 \theta\right), \\
& A_{d}=\omega^{2} r\left(\cos \theta+\frac{r}{l} \cos 2 \theta\right) .
\end{aligned}
$$

Unbalance mass of the cylinder piston crank mechanism consists of three parts: mass of the rotational crank, the connecting rod, and the reciprocating piston. Considering mass of crank as $M_{a}$ while the assembly of connecting rod and piston is $M_{b}$, the unbalance forces can be divided into two parts: rotational unbalance force $F_{a}$ caused by the crank and reciprocating unbalance force $F_{b}$ caused by the assembly

$$
\begin{aligned}
& F_{a}=-M_{a} \omega^{2} r \\
& F_{b}=-M_{b} \omega^{2} r\left(\cos \theta+\frac{r}{l} \cos 2 \theta\right) .
\end{aligned}
$$

The negative sign indicates the force is in the opposite direction with the acceleration. According to the working principle of the engine, the vibration output of the engine mainly depends on the reciprocating force of the assembly [12].

2.2. Inertia Torque. According to mechanics principle, the inertia torque on the crank from a single piston is

$$
T=F_{b} \cdot L \cdot \tan \varphi,
$$

where $L$ refers to moment arm from crank center and

$$
L=r \cos \theta+l \cos \varphi=r+l-X_{d} .
$$

That is,

$$
L \cong l-\frac{r^{2}}{4 l}-r \cos \theta+\frac{r^{2}}{4 l} \cos 2 \theta
$$

Also, using trigonometric transformation and disregarding all terms proportional to the second or higher powers of $r / l$, the final expression of the inertia torque can be presented as

$$
T=\frac{1}{2} M_{b} \omega^{2} r^{2}\left(\frac{r}{2 l} \sin \theta-\sin 2 \theta+\cdots\right) .
$$

As can be seen from the expression, the inertia torque acts in the rotational direction. The individual terms of the equation present the first-order, second-order, third-order, and some higher order harmonic components for the engine vibration.

Idle vibration of a forklift vehicle involving a four-cylinder, 4-stroke engine always happens at low engine speed from 720 to $840 \mathrm{rpm}$. Since the piston pairs are working along opposite directions, the first-order inertial forces can be well balanced. Meanwhile, the two descending pistons travel further than the ascending pairs since the movement of the connecting rod accelerates the descending pistons while delaying the ascending ones, producing a resultant force that

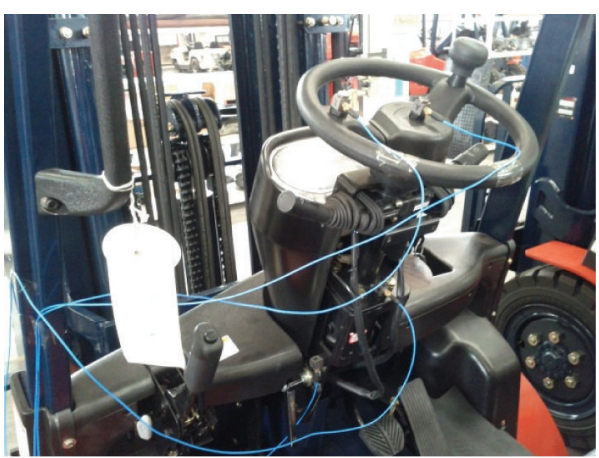

FIgURE 3: Measurements for the steering system.

varies periodically twice per crankshaft revolution. Also, the second-order forces and their multiples occur due to the cyclic change of the crank angle. That is, the frequency of unbalance disturbances is correlated with the number of cylinders and stokes. In this study, the idle vibration analysis is usually conducted in the $24 \mathrm{~Hz}$ to $28 \mathrm{~Hz}$ frequency range, equating to the engine speed for second-order firing.

\section{Field Test of the Idle Vibration of the Steering System}

In order to fully extract the idle vibration character information of the steering system, field tests were carried out. The acceleration transducers were reasonably distributed through vibration transmission path, which is shown in Figure 3. The directions of the measurement at the steering system are taken along three principal axes of the vehicle. $X$ and $Y$ define the plane of the steering wheel, where $y$ axis is directed towards the left of the vehicle and $x$ axis is the tangent of the steering wheel directed towards the front of the vehicle. Meanwhile, $z$ axis is perpendicular to the $X-Y$ plane.

Figure 4 shows the RMS value of vibration acceleration of the wheel steering in each direction changing with the engine speed. As is shown in the figure, vibration amplitudes of the $Y$ and $Z$ directions are much larger at speed of about $770 \mathrm{rpm}$ than other conditions. Figure 5 represents the waterfall curve of the vibration amplitude in $Y$ direction applying the Fourier algorithm to equal-interval sampling vibration data. The order tracking analysis reveals that the second-order firing frequency (25.7 Hz, equating to the engine speed of $780 \mathrm{rpm}$ ) has great contribution to the idle vibration of the wheel system.

Working under idle condition (engine speed of $750 \mathrm{rpm}$ ), the second-order firing frequency of the engine turns out to be $25 \mathrm{~Hz}$. When the steering system's operating modal frequency is close to the excitation frequency, resonance of the steering system happens. The first- and second-order modes have great contribution to the idle vibration of the forklift vehicle.

\section{Finite Element Analysis of the Steering System}

With the development of computer technology, computer aided engineering has already become important ways of 
TABLE 1: Modal frequency and shapes of steering system.

\begin{tabular}{lcccc}
\hline Order & Modal shape & $\begin{array}{c}\text { FE modal } \\
\text { frequency }\end{array}$ & $\begin{array}{c}\text { Experimental } \\
\text { modal frequency }\end{array}$ & $\begin{array}{c}\text { Relative } \\
\text { difference }\end{array}$ \\
\hline 1 & Steering wheel's left and right movement & 24.01 & 25.74 & $7.2 \%$ \\
2 & Steering wheel's pitching motion & 27.63 & 28.24 & $2.2 \%$ \\
3 & Steering system's left and right movement & 76.91 & - & - \\
\hline
\end{tabular}

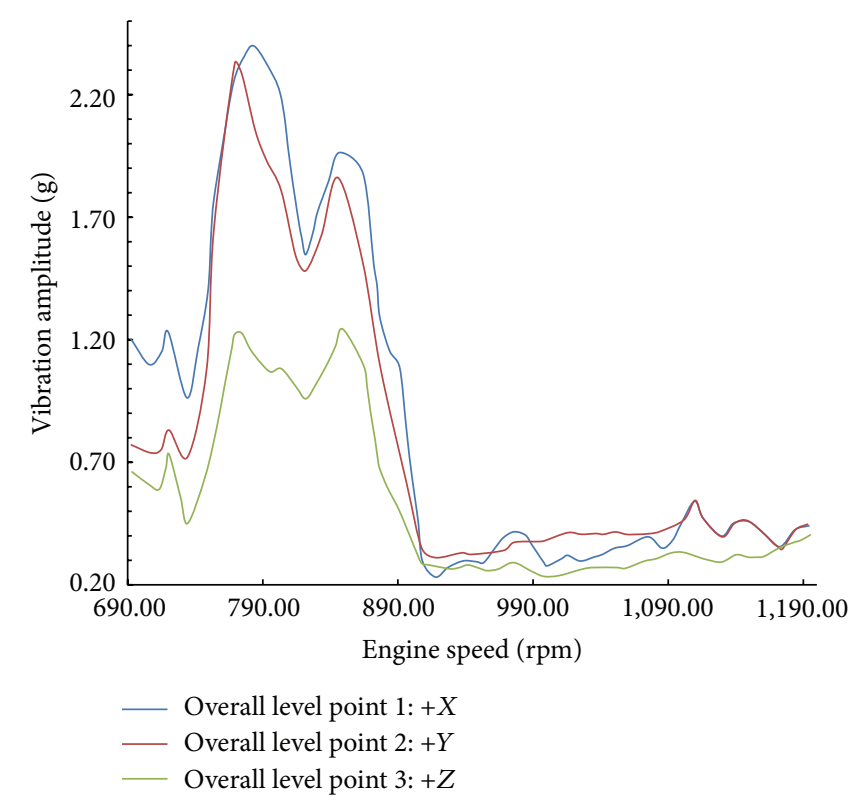

FIGURE 4: RMS value of vibration acceleration.

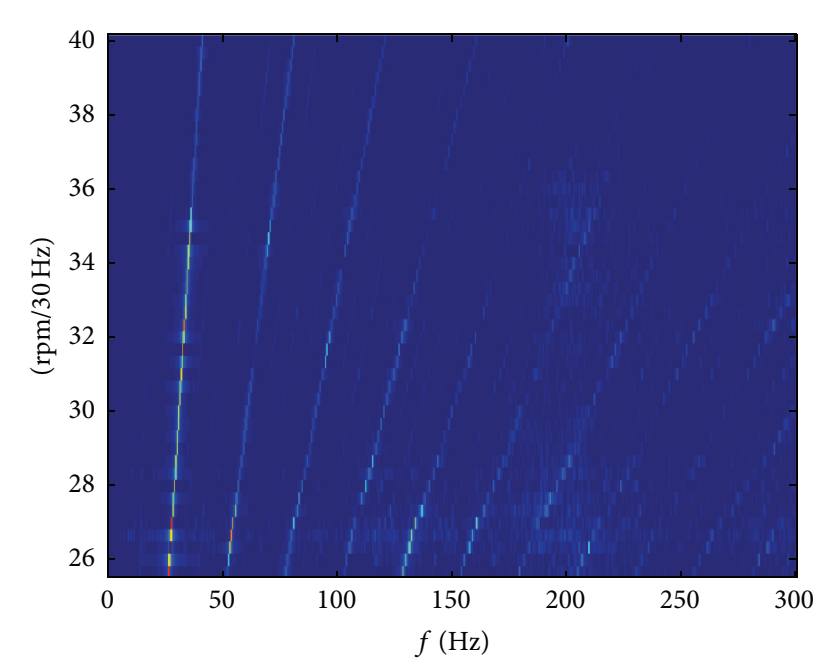

FIGURE 5: Waterfall curve of the vibration amplitude.

modern mechanism research and plays important roles in feasibility verification, engineering design, and preferred plan selection. In the field of mechanism characteristic analysis especially, to explore the robustness and performance of mechanical components and assemblies, computer simulation with the utilization of CAE software has been extensively developed.

In order to assess the dynamic characteristics of the structure, finite element modal analysis was performed to determine the natural frequencies, mode shapes, and frequency constraints of the structure. Through modal analysis method, we can find the major modal characteristics of the steering system in a particular frequency range, thereby understanding the actual vibration response under the engine excitation.

Pro/ENGINEER is the industry's first successful rulebased parametric 3D CAD modeling system. The parametric modeling approach uses parameters, dimensions, features, and relationships to capture intended product behavior and create a recipe which enables design automation and the optimization of design and product development processes. With the help of "mechanism," which is a modular of Pro/ ENGINEER, kinematic and dynamic analysis and simulations can be carried out in order to look into the virtual movement of the mechanism, thus getting the kinematics parameters of the object throughout the process.

The steering system consists of fore plate, mounting plate, support plate, steering column, and steering wheel. The virtual prototype of the system is shown in Figure 6(a). Then, cleaning up the geometry, defining the material, setting the boundary constraints and meshing the model. Meshing has a very important role in modeling which will directly affect the accuracy of the computational efficiency; unreasonable meshing would affect the constringency effect of the solution. Automatic mesh of ProE using $\mathrm{H}$-adaptive method is subdivision mesh again in the high stress gradient position based on the convergence criteria stipulated beforehand to solve the computation convergence problem. According to the structural characteristics and load, solid 187 is selected as element type; the finite element model after automatic mesh division has been established shown in Figure 6(b), including 2711 nodes, 12842 edges, 17638 faces, and 7529 solid elements.

Because we just care about the idle frequency $(24 \sim 28 \mathrm{~Hz})$, only the former three-order modes are shown in Figure 7; the frequencies are shown in Table 1. The first-order modal frequency is close to the idle frequency, so is the second one.

During the process of modeling the FEM model, some mechanical features have been reasonably predigested. Using the hammering method, we can also get the modal parameter by field tests in order to verify the FEM analysis. As shown in Figure 8, acceleration sensors were arranged to collect vibration acceleration signals of steering system; the mode parameters extracted are shown in Table 1. 


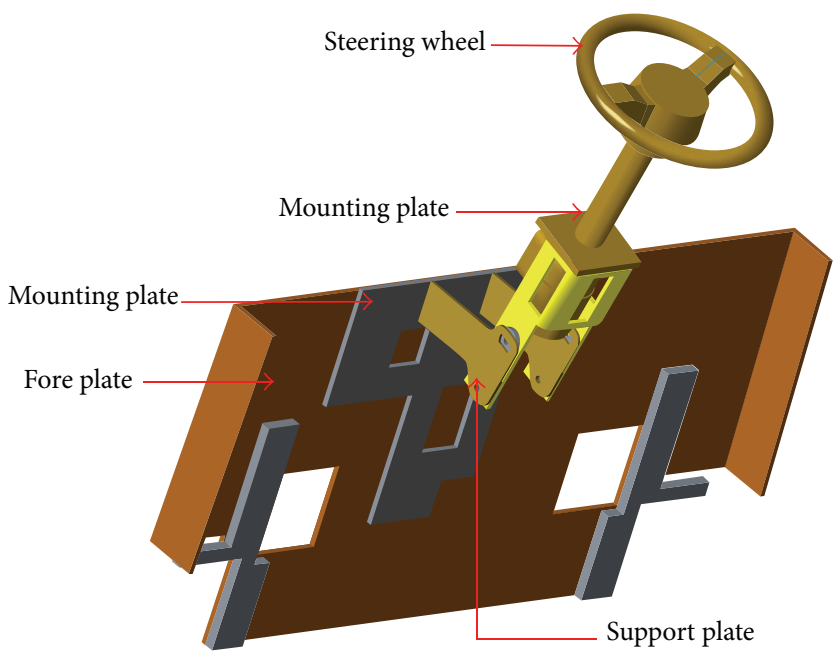

(a)

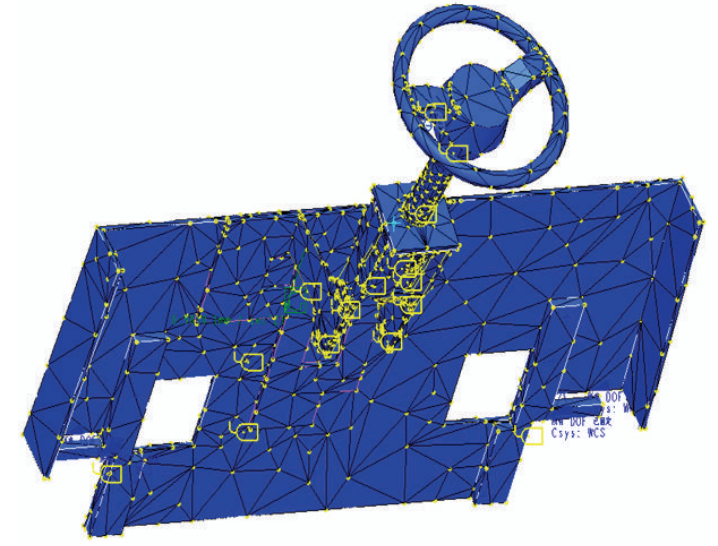

(b)

FIgURE 6: (a) Virtual prototype of the steering system. (b) Finite element modal of the steering system.

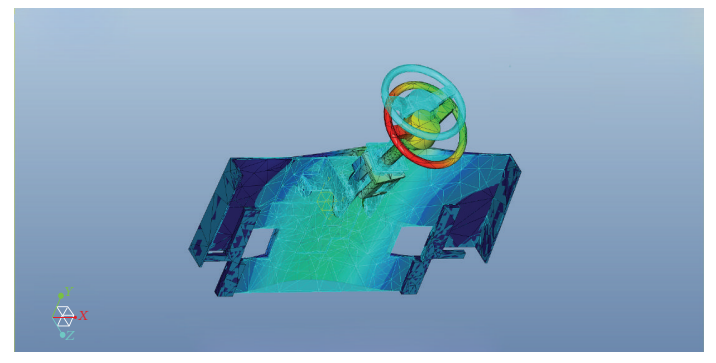

(a) Mode 1

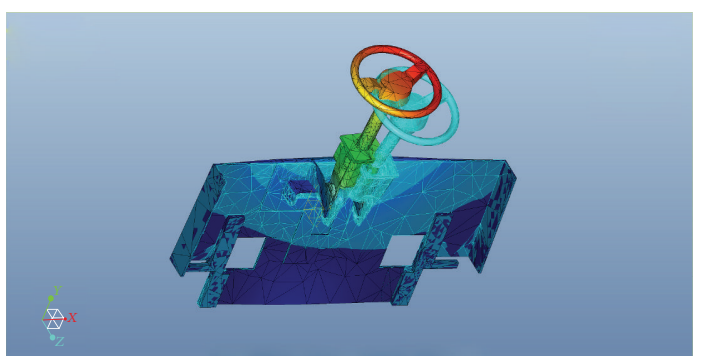

(b) Mode 2
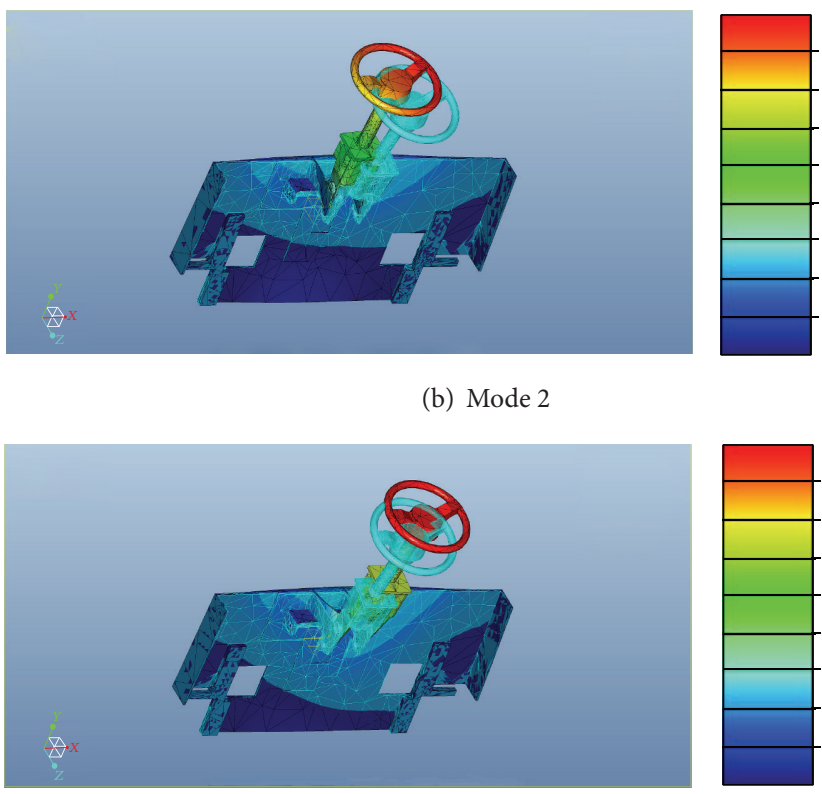

$1.000 e+00$ $8.889 e-01$ $7.778 e-01$ $6.667 e-01$

$5.556 e-01$ $4.444 e-01$ $3.333 e-01$

$2.222 e-01$

$1.111 e-01$

$0.000 e+00$

(c) Mode 3
$1.000 e+00$

$8.889 e-01$

$7.778 e-01$

$6.667 e-01$

$5.556 e-01$

$4.444 e-01$

$3.333 e-01$

$2.222 e-01$

$1.111 e-01$

$0.000 e+00$

FIgUre 7: Modal shapes of the steering system. 


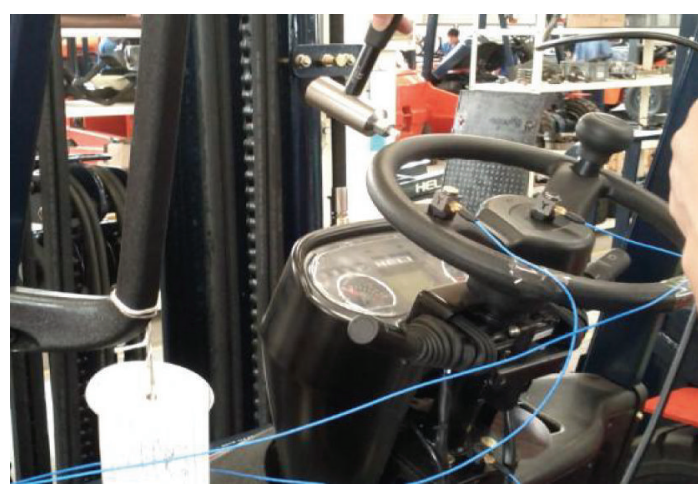

FIGURE 8: Arrangement of measure points of hammer excitation test.

\section{Improvements of Steering System's Idle Vibration}

In order to relieve vibration, most effective resolutions are vibration isolation, vibration attenuation, and resonance avoiding. Based on the FEM analysis and the field tests, it is found that the main reason for the steering system's idle vibration of the forklift vehicle is the resonance excited by the engine. Since the modal frequency of the steering system is mainly decided by the mass and stiffness, the optimization of the structure can be acquired by reasonably distributing the mass and stiffness of the steering system, so as to set the modal frequencies out of the resonance frequency range, suppressing the idle vibration.

As is mentioned above, the modals of the steering system in the vicinity of the resonance frequency were very close to one another; successive resonance will also occur in low engine speed, which was violence against the stable mechanical structure. From the engineering point of view, the interval between the two modals should sufficiently avoid the resonance range from the engine idle frequency. Considering the original system structure, some measures were carried out to reduce the first modal frequency while increasing the second modal frequency. During the optimization process, some algorithms were adopted, especially Sequence Quadratic Program (SQP) algorithm.

5.1. Sequence Quadratic Program (SQP) Algorithm. Sequential quadratic programming method is considered as the most efficient methods for solving NLPs

$$
\begin{array}{ll}
\min & f(x) \quad x \in \mathbb{R}^{n} ; \\
\text { s.t. } & h_{i}(x)=0 \quad i \in E=\{1,2, \ldots, m\}, \\
& g_{j}(x) \geq 0 \quad j \in I=\{m+1, \ldots, n\},
\end{array}
$$

where $E, I$ are index set with equality and inequality constraints, $m, n$ equal to the number of constraints. Assume that $f, h$, and $g$ are defined on all of $\mathbb{R}^{n}$ and two times continuously differentiable functions. The SQP method computes a correction for a given approximation $x^{k}$ of a solution of NLP by solving a QP problem; thus inconsistent QP problems are solved in the SQP method

$$
\begin{array}{ll}
\min & \nabla f\left(x_{k}\right)^{T} d+\frac{1}{2} d^{T} H_{k} d, \\
\text { s.t. } & \nabla h_{i}\left(x_{k}\right)^{T} d+h_{i}\left(x_{k}\right)=0 \quad i \in E, \\
& \nabla h_{i}\left(x_{k}\right)^{T} d+h_{i}\left(x_{k}\right) \geq 0 \quad i \in I .
\end{array}
$$

Here $H_{k}$ is chosen symmetric positive semidefinite matrix; given $x_{k}$ as a guess for (local) solutions $x^{*}$ and $H_{k}$, the correction $d_{k}$ is computed as the solution of the equality constrained subproblem. Then one lets $x_{k+1}=x_{k}+\sigma_{k} d_{k}$ for suitably chosen stepsize $\sigma_{k}>0 . \sigma_{k}$ is computed by a descent test for so-called merit function.

The solving steps of SQP areas follow.

(1) Setting the initial points $x_{0}, a_{k}$, and $H_{k}$.

(2) Solving the QP problem, determining the value of $\lambda_{k}$ and $d_{k}$.

(3) Determining the value of $\sigma_{k}$.

(4) Checking the convergence of the solution.

5.2. Redudeterminection of the First Modal Frequency. Compared with the modal analysis, the lateral stiffness of the steering system was important to the first modal frequency. As is shown in Figure 9, the origin design of the support plate and pedal bracket was solder connection. Although this connection ensured enough stiffness to perform less vibration amplitude under the same stress, the design increased the frequency of the system. As a result, the first modal frequency entered the resonance frequency range, intensifying vibration. To avoid the happening of sympathetic vibration, one idea is decreasing the frequency. Based on the mechanical system design, the solder parts of the support plate and pedal bracket were cut to be separated. This measure was equivalent to wiping out the lateral stiffener while the thickness of the support plate ensured enough lateral stiffness to load relative stress. FEM analysis showed that, through this separation, the first modal frequency of steering system has changed to be $19.73 \mathrm{~Hz}$, which verified the rationality of this improvement.

5.3. Increase of the Second Modal Frequency. The second modal frequency of steering system was dependent on the longitudinal stiffness and finite element analysis was aimed at structure optimization, redistribution of the mass, and stiffness. Before optimization, global sensitivity analysis should be carried out first in order to confirm high sensitivity parameters and their variables value sector. As is shown in Figure 10, the second modal frequency was sensitive to thickness of fore plate $\left(D_{1}\right)$ and mounting plate $\left(D_{2}\right)$, followed by height of mounting plate $\left(D_{3}\right)$ and length of mounting plate $\left(D_{4}\right)$, which were set to be optimization parameters. The modal frequencies were desired to be out of the resonance range, so the second modal frequency must be more than 30 , as the objective function min $f(x)$. Based on Pro/mechanic module, SQP algorithm mentioned above was used. 


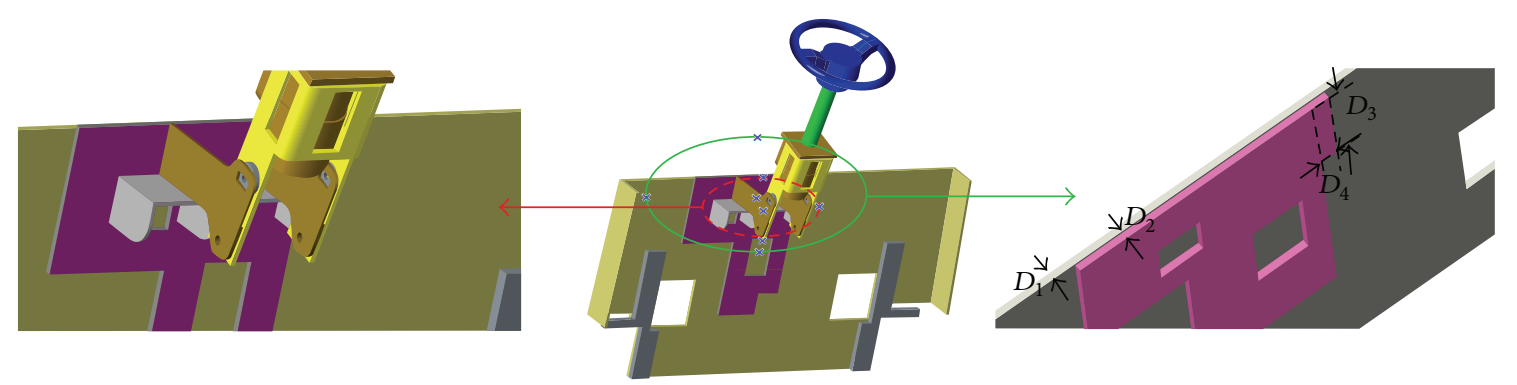

FIGURE 9: Main construction of the steering system.
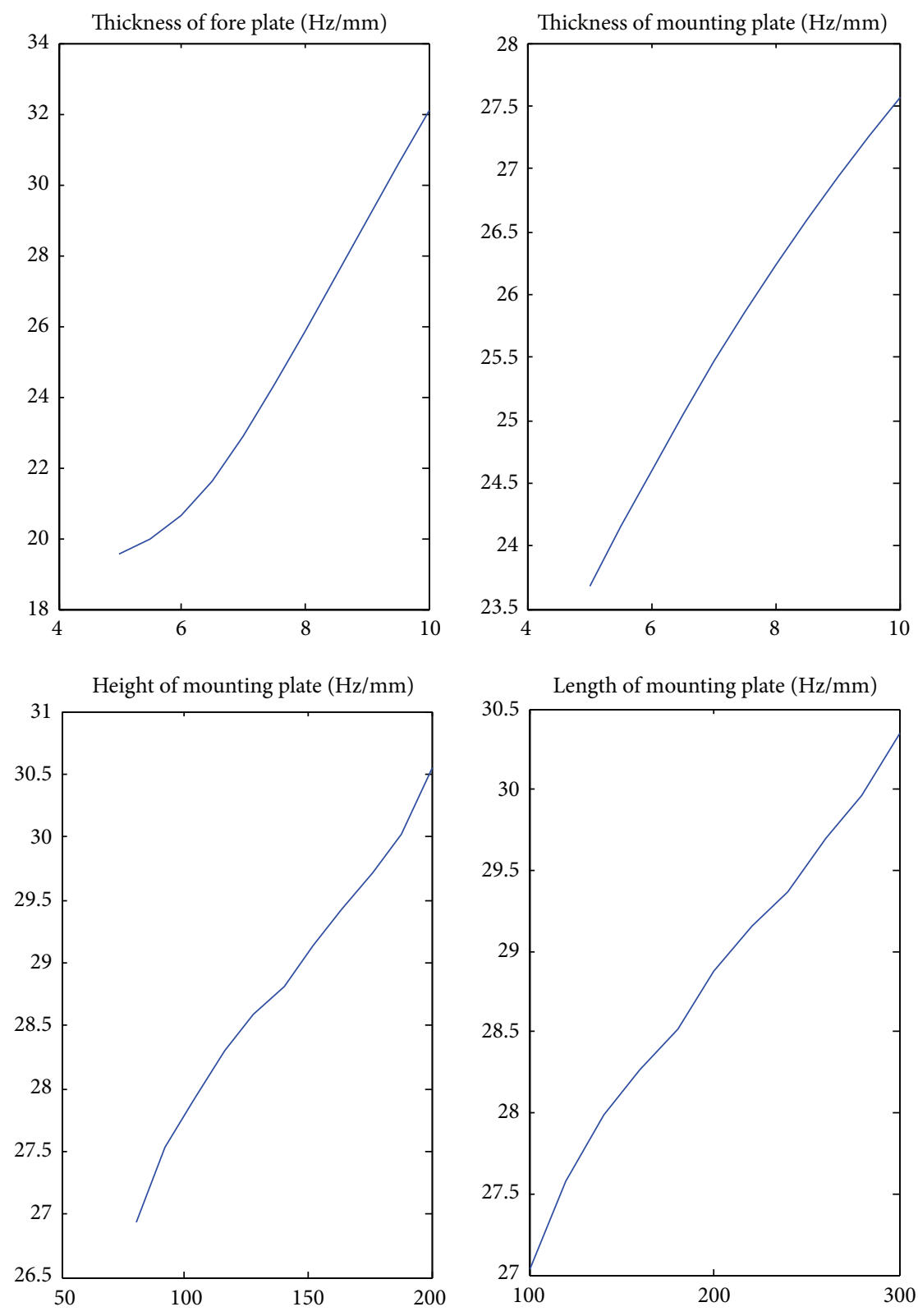

FIGURE 10: Sensitivity curves of main parameters. 
TABLE 2: Value of the optimal parameters $/ \mathrm{mm}$.

\begin{tabular}{lcccc}
\hline Design variables & $D_{1}$ & $D_{2}$ & $D_{3}$ & $D_{4}$ \\
\hline Initial value & 7 & 7 & 150 & 200 \\
\hline Optimal ranges & $(-2,3)$ & $(-2,3)$ & $(-70,50)$ & $(-100,100)$ \\
\hline Optimal value & 8.1 & 8.3 & 180.1 & 250.3 \\
\hline Adjusted optimal value & 8 & 8 & 180 & 250 \\
\hline
\end{tabular}

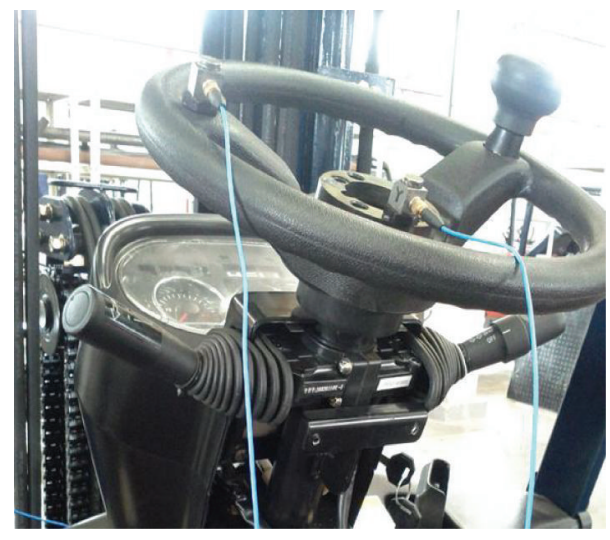

FIGURE 11: Arrangement of acceleration sensors.

Actually, only these parameters above can be optimized according to the mechanical model, and these parameters can be only changed in the optimal range shown in Table 2. Otherwise, it will affect the installation and cooperation of this structure. So the actual sizes of the forklift steering wheel assembly are selected as the initial value and then the parameters are optimized in the optimal ranges. After iterative computation 9 times, optimized scheme was calculated. Defining the convergence as $1 \%$, initial values and optimal values of the parameters are listed in Table 2. Considering the components processing technology and the machining cost, the optimal parameters are appropriately adjusted.

As is mentioned above, to relieve steering system's vibration, two measures were presented. Through the improvements, the first modal frequency of the system should be decreased while the second one went to the opposite. Based on the modified structure, finite element analysis was carried out. Results showed that the first modal frequency has changed to $19.73 \mathrm{~Hz}$, which was $17.82 \%$ less than the former one. The second modal frequency has changed to $30.13 \mathrm{~Hz}$, which was $9.05 \%$ higher than the former one. The optimized frequencies were far enough from the engine idle frequency for the second-order firing of the internal combustion engine.

Idle experiments were also carried out to verify the improving effect of the structural optimization. According to 〈〈GB/T 14790.1-2009 Mechanical vibration: Measurement and evaluation of human exposure to hand-transmitted vibration $\rangle\rangle$. As is shown in Figure 11, acceleration sensors were arranged on the steering wheel and idle vibration acceleration amplitudes of the steering system were collected. Compared with the original structure, vibration amplitude of steering system with improvements had substantially declined from $3.36 \mathrm{~g}$ to $0.98 \mathrm{~g}$, and steering system's idle vibration was effectively suppressed.

\section{Conclusion}

In this paper, we present a research on the performance deficiencies about vibrations of steering system while the forklift vehicle is working under idle condition. Acceleration transmitted to the driver's steering wheel was chosen to be the representation of the transmitted vibration. The idle vibration signals were collected to extract characteristics. Then, the FEM analysis of the steering system was conducted, compared with the vibration signals. So as to get the contribution of each design parameter, sensitivity analysis was also implemented to achieve steering system design optimization for idle vibration reduction. Experiments were carried out to verify the effectiveness of the reconfiguration of the characteristic modes. This optimal design is not a case study; it can be also applied to other objects that experience vibration restrictions, which has a bright future in our engineering implementation.

\section{Conflict of Interests}

The authors declare that there is no conflict of interests regarding the publication of this paper.

\section{Acknowledgments}

This work was partially supported by Natural Science Foundation of Anhui Province (1308085QE92), Key University Science Research Project of Anhui Province (KJ2014A203), and The Excellent Young Talents Support Project in Universities of Anhui Province.

\section{References}

[1] A. Behar and S. Libich, "Whole body vibration measurements of forklift truck drivers," Canadian Acoustics, vol. 33, no. 4, pp. 39-42, 2005.

[2] K. C. Kim, I. H. Choi, and C. M. Kim, "A study on the advanced technology analysis process of a steering system for idle vibration," Tech. Rep. 01-2339, 2007.

[3] A. P. Cann, A. W. Salmoni, P. Vi, and T. R. Eger, "An exploratory study of whole-body vibration exposure and dose while operating heavy equipment in the construction industry," Applied Occupational and Environmental Hygiene, vol. 18, no. 12, pp. 999-1005, 2003.

[4] W.-K. Shi, G.-M. Wu, Z.-Y. Chen, L.-M. Gui, F.-X. Guo, and D.G. Fang, "Steering wheel's shaking control under idling operation of a car engine," Journal of Vibration and Shock, vol. 32, no. 8, pp. 189-193, 2013.

[5] M. Shariyat and P. Djamshidi, "Minimizing the engine-induced harshness based on the DOE method and sensitivity analysis of the full vehicle NVH model," International Journal of Automotive Technology, vol. 10, no. 6, pp. 687-696, 2009.

[6] M. L. Xu and G. N. Xu, "Vibration study on forklift truck based on the constraint-rigid-flexible coupling system," Journal of Mechanical Engineering, vol. 47, pp. 89-94, 2011. 
[7] H. B. Parker, J. U. Lee, and J. K. Suh, "Design of high stiffness steering system for idle vibration," in Proceedings of the KSME Spring Conference, no. 97380112, 1997.

[8] K. C. Kim, I. H. Chio, and C. M. Kim, "A study on the development process of a body with high stiffness," SAE Paper 200401-2464, 2005.

[9] T. J. Royston and R. Singh, "Optimization of passive and active non-linear vibration mounting systems based on vibratory power transmission," Journal of Sound and Vibration, vol. 194, no. 3, pp. 295-316, 1996.

[10] R. Singh, "Dynamic design of automotive systems: engine mounts and structural joints," Sadhana, vol. 25, no. 3, pp. 319330,2000

[11] J. D. Jia, J. Chen, and Q. Q. Gao, "Test analysis and control research on the idling resonance of a certain vehicle," Transaction of the Chinese Society for Agricultural Machinery, vol. 40, pp. 40-43, 2009.

[12] K. C. Kim and C. M. Kim, "Analysis process of a steering system using a concept model for idle vibration," International Journal of Automotive Technology, vol. 9, no. 3, pp. 337-346, 2008. 


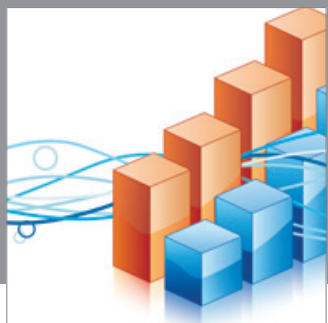

Advances in

Operations Research

mansans

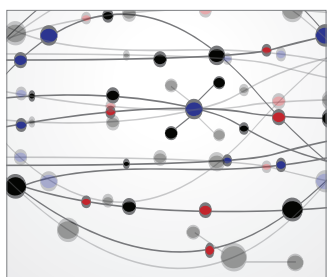

The Scientific World Journal
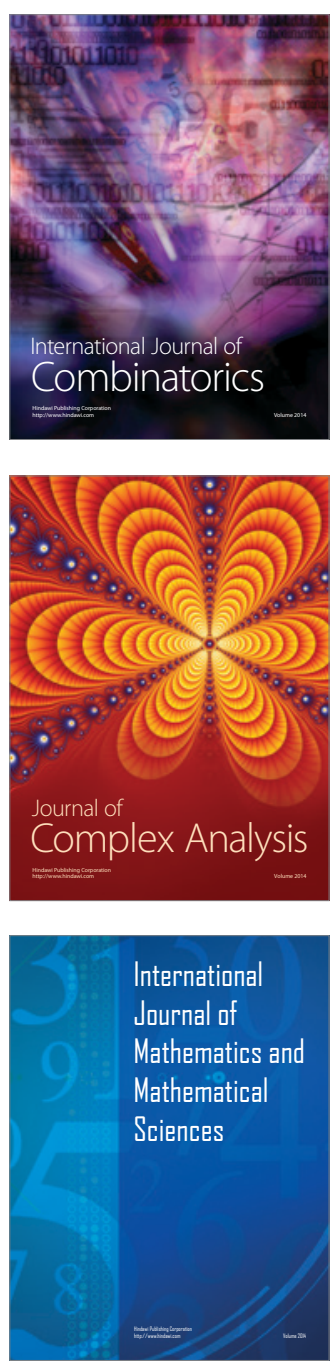
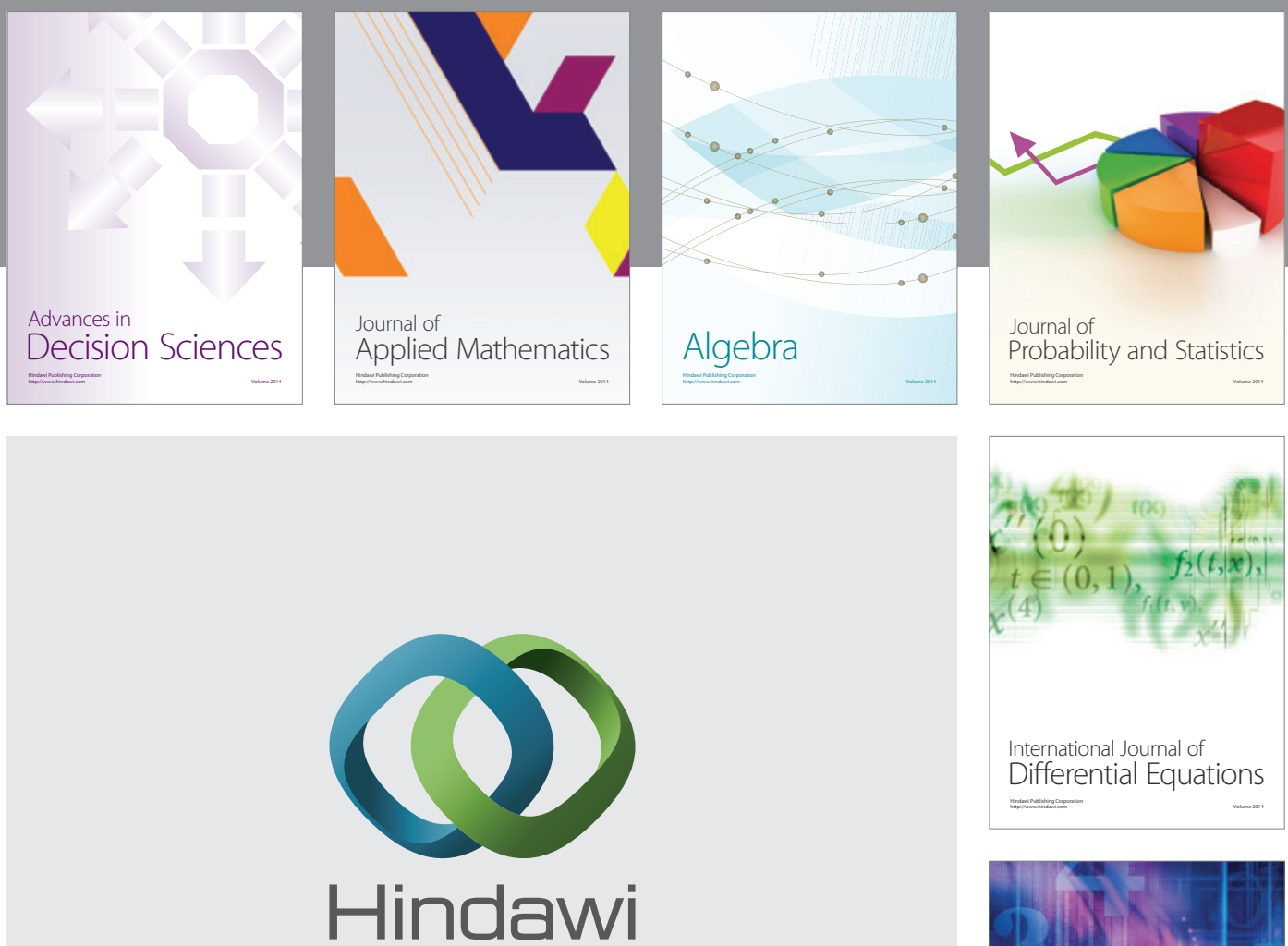

Submit your manuscripts at http://www.hindawi.com
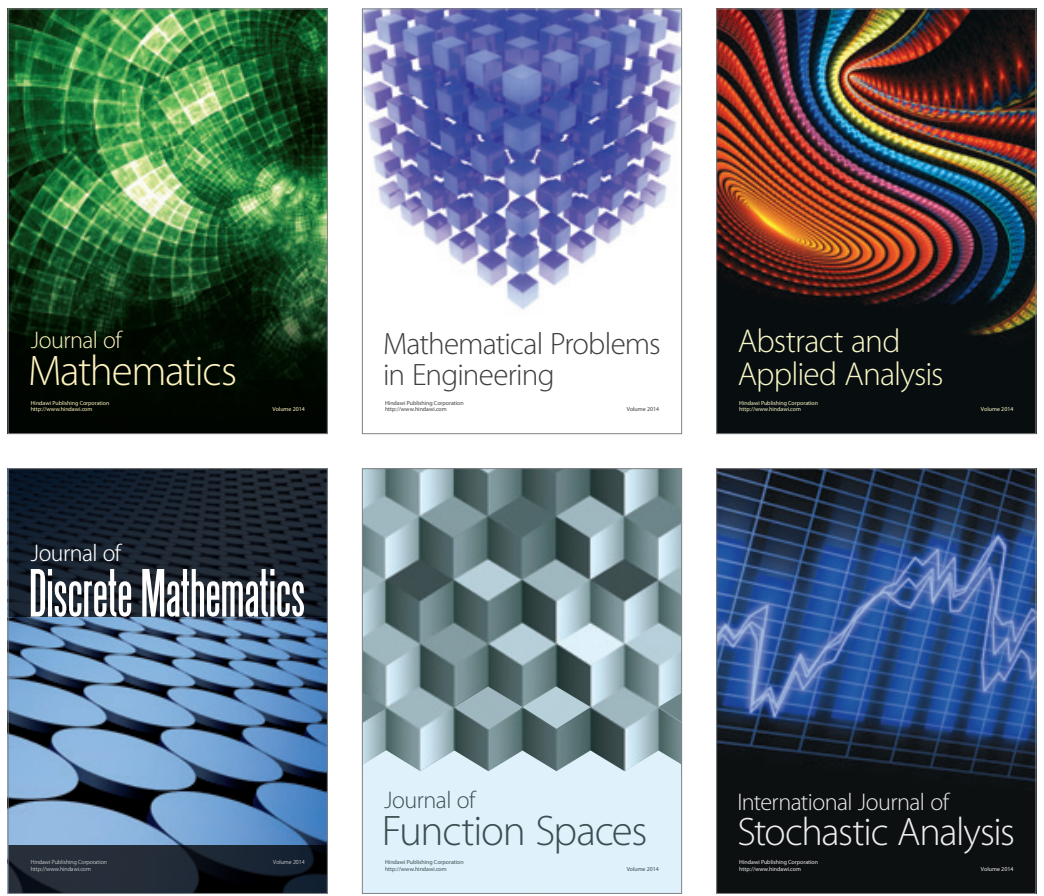

Journal of

Function Spaces

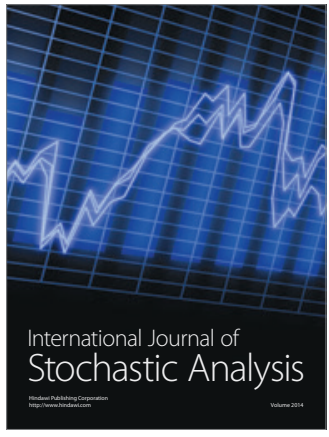

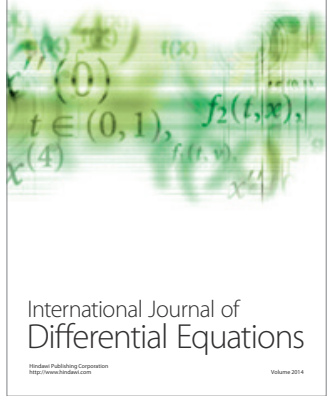
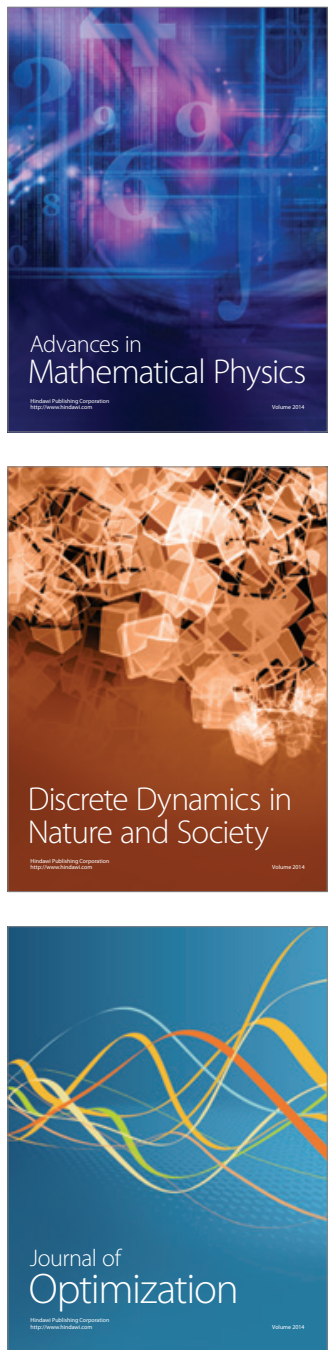